\title{
Dynamic Handoff Threshold Algorithm Using Mobile Speed for WLAN Utilization Improvement in 3G-WLAN Integrated Networks*
}

\author{
JangSub Kim, HoJin Shin, and DongRyeol Shin \\ School of Information and Communication Engineering, \\ Sungkyunkwan University, \\ 300 ChunChun-Dong, JangAn-Gu, Suwon, Korea \\ \{jangsub, hjshin, drshin\}@ece.skku.ac.kr
}

\begin{abstract}
In 3G-WLAN integrated networks, for high data-rate WLAN (Wireless LAN) network the user wants to maintain the WLAN connection as long as possible and then switch to the overlaying $3 \mathrm{G}$ cellular data service dynamically. Thus, we propose a new dynamic threshold for seamless vertical handoff, are used to more long maintain the WLAN connection compared to fixed threshold, thus total WLAN usability is increasing. We present the design architecture of the proposed method and evaluate its performance in a network environment.
\end{abstract}

\section{Introduction}

$3^{\text {rd }}$ Generation cellular and WLANs will complement each other to provide ubiquitous high-speed wireless Internet connectivity to mobile users. Therefore, it is important to consider dual mode users roaming in between $3 \mathrm{G}$ cellular and WLANs. In order to provide a convenient access of both technologies in different environments, interworking [1] of the two networks are regarded as a very important work.

In this paper, we propose a new mechanism for obtaining link layer indication is dynamically transported to the upper layer (network layer). The dynamic threshold as function of mobile node speed be used to information of triggers for low latency MIPv4 [2] and fast MIPv6 [3]. To extend the WLAN service time we will find the optimal value for dynamic threshold to relate with RSS (Received Signal Strength) and mobile speed. The fixed threshold value which is not considered user speed cannot be fully used to maintain WLAN service as long as possible. But the proposed dynamic threshold which is adapted user speed has more advantage than general mechanism. We show improvement of the utilization of during WLAN service.

In Section 2, problems are formulated, and core part of algorithmic for dynamic threshold. Simulations are performed in Section 3 to validate the proposed approach. Finally, the summary of the result are presented in the conclusion section.

\footnotetext{
This research is supported by the ubiquitous Autonomic Computing and Network Project, the Ministry of Information and Communication (MIC) 21st Century Frontier R\&D Program in Korea.
} 


\section{Handoffs Optimization}

This section presents the vertical handoff triggers analyses. In case of fixed threshold value to trigger, the handoff from $3 \mathrm{G}$ cellular network to WLAN carried out irrespective of mobile node speed. When a mobile node moves from one network to another, if the preparation time of fast handoff is larger than WLAN sojourn time related to mobile node speed, the handoff failed and occurred the packet loss. If the mobile node speed is too slow in case of fixed threshold value, instead, handoffs are triggered too late and thus WLAN service time is reduced. Thus in this paper, we have considering dynamic threshold as function of mobile node speed. In this case the mobile node speed is too slow, threshold is alter to small value, instead, handoffs are triggered too early and thus WLAN service time is increasing and total utilization is increasing.

To find the optimal dynamic threshold values $\left(T_{H d}\right.$ and $\left.T_{L d}\right)$ we set up a test to relate RSS and user speed. Generally, the channel propagation model used for RSS is given by [4]. For analysis simplicity, we are assumed the RSS on WLAN link shown as Fig. 1.

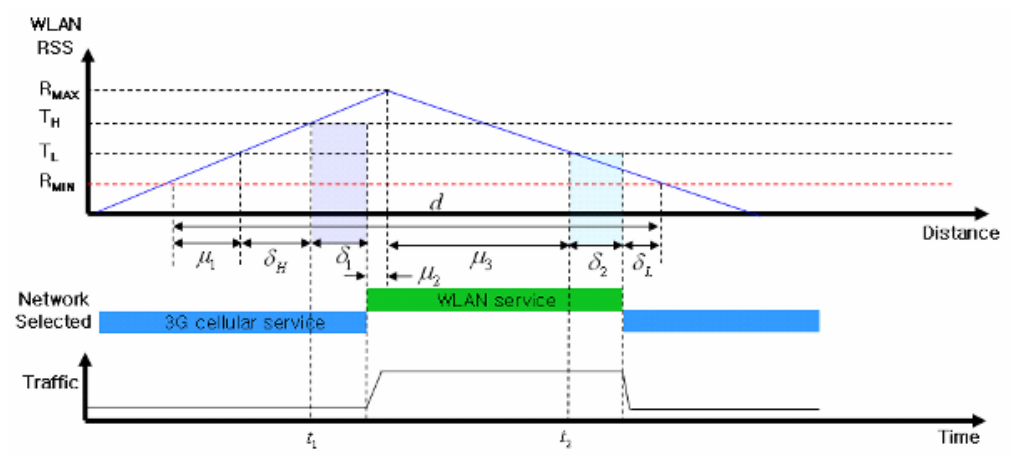

Fig. 1. A vertical handoff from $3 \mathrm{G}$ cellular to WLAN

We use the following variables to determine the vertical handoff analysis. Where $\mathrm{R}$ is received signal strength, $d$ is diameter of WLAN coverage, $\delta_{1}$ and $\delta_{2}$ are handoff prepare time from $3 \mathrm{G}$ to WLAN and from WLAN to $3 \mathrm{G}$, respectively. Specifically, it shows the WLAN signal observed by the client over time. At $t_{l}$, when the signal strength exceeds the threshold, $T_{H}$, the client will attempt to use the WLAN airlink. Similarly at time $t_{2}$, when the signal strength drops below the threshold, $T_{L}$, the client will revert to the $3 \mathrm{G}$ airlink. Two thresholds, $T_{H}$ and $T_{L}$, are used to avoid unnecessary handoffs that can result in poor connection. In this paper, two threshold is using to instead of dwell timer.

We omit the detailed derivation of dynamic threshold values for lack of space, thus we show the resulting and proposed perspective of that. The user speed is related with the slope of the RSS. We have to find the dynamic threshold satisfied the following equation.

$$
T_{H d}=\frac{R(t+1)-R(t)}{T(t+1)-T(t)} \cdot \delta_{H}, T_{L d}=\frac{R(t+1)-R(t)}{T(t+1)-T(t)} \cdot\left(\delta_{2}+\delta_{L}\right)
$$


The procedure is now concerned with the $T_{H d}$ and $T_{L d}$ in which can be written as a function of velocity $(V)$, and hence finding the value of $T_{H d}$ and $T_{L d}$. If shadowing fading is existed, the slope of the RSS ( $\mathrm{dBm}$ per unit second or $\mathrm{dBm}$ per unit meter) be calculated to use smoothing method, following by

$$
\nabla R_{t+1}=\alpha \nabla R_{t+1}+(1-\alpha) \nabla R_{t}
$$

Thus, as we are considering the previous quantity, we avoid to abrupt change quantity in the WLAN environments. ais the memory factor.

The proposed perspective of dynamic threshold calculation procedure is shown in Fig. 2. To calculate the dynamic threshold, firstly, a MN has to scan the AP signal. If the RSS is larger than $R_{M I M}$, the MN has to measure a change quantity of the RSS per unit time. Because the change quantity of that is proportion to the mobile velocity, we can be estimated the mobile velocity. At this time, if the GPS or ToA information is available, the mobile velocity is correction for the higher prediction accuracy. Using (2), we calculate the dynamic threshold. This dynamic threshold is sensitivity to the mobile velocity. According to the MN velocity, the dynamic threshold becomes altered to extend the WLAN service time more than $3 \mathrm{G}$ networks. If the RSS is larger than $T_{H d}$ or is smaller than $T_{L d}$, then the seamless vertical handoff be started. We make the L3 handoff as closer as the L2 handoff, then the MN has low latency.

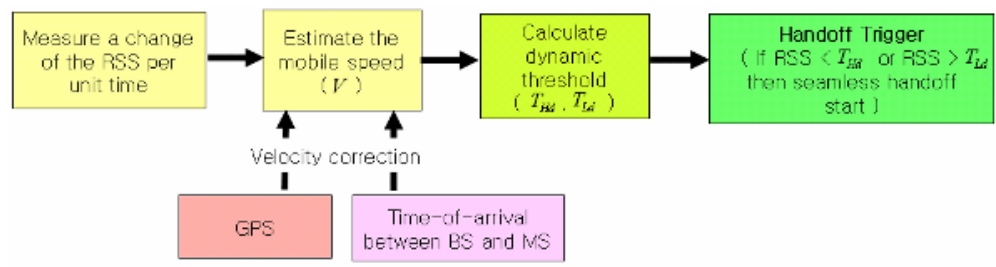

Fig. 2. The procedure of the dynamic threshold calculation

\section{Numerical Analysis}

The proposed procedure is tested with a number of numerical examples for the overlaid structure. The WLAN constitutes the lower layer of the two-layer hierarchy. The WLAN are overlaid by a large $3 \mathrm{G}$ networks, which forms the upper cell layer. In our system, mobile node are traversing the coverage are of the WLAN and 3G network. The diameter of the WLAN is assumed to be $d=100 \mathrm{~m}$. The downward (from $3 \mathrm{G}$ to WLAN) vertical handoff preparation time and the upward (from WLAN to $3 \mathrm{G}$ ) vertical handoff preparation time are assumed to $\delta_{1}=500 \mathrm{~ms}$ and $\delta_{2}=500 \mathrm{~ms}$, respectively. And we are assumed to $\delta_{H}=1 \mathrm{sec}$ and $\delta_{L}=1 \mathrm{sec}$. From Fig. 1, the WLAN service time is given by

$$
W L A N_{\text {service time }}=\mu_{2}+\mu_{3}+\delta_{2}
$$

Fig. 3 illustrate the WLAN service time as user speed. As the figure indicated, the WLAN service time of the proposed method is longer than the fixed threshold below 
the user speed with $30 \mathrm{~m} / \mathrm{sec}$. Thus, the dynamic threshold to low speed user have add benefit that the WLAN service time is a more long than fixed threshold. In the mobile velocity at $1 \mathrm{~m} / \mathrm{sec}$, the WLAN service time is extended to 1.7 times, compared to fixed threshold. Thus, we have to apply the dynamic threshold in the mobile velocity at below $30 \mathrm{~m} / \mathrm{sec}$. Our simulation is easily extended to the real received signal model for longer the WLAN service time.

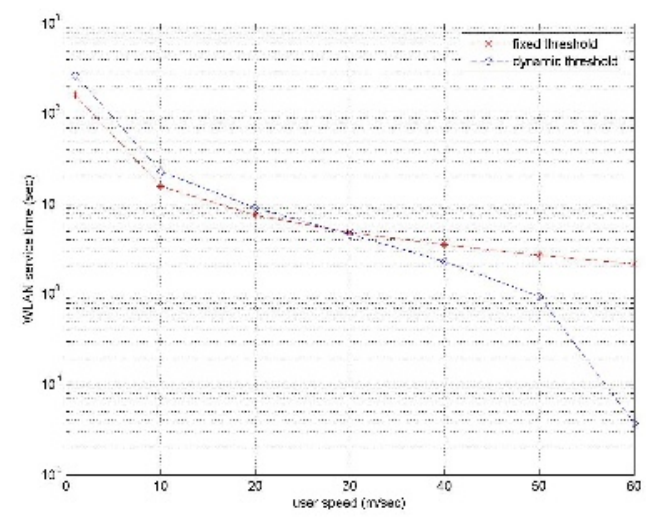

Fig. 3. WLAN service time as user speed

\section{Conclusion}

We have proposed a dynamic threshold for slow moving user and simply analysis the dynamic threshold in order to improve the utilization of WLAN more than $3 \mathrm{G}$ cellular network. The analysis results show the dependency of the WLAN utilization improvement upon the dynamic threshold, $T_{H d}$ and $T_{L d}$. The dynamic threshold has shown to be an important system parameter that the system providers should determine to produce better utilization improvement.

\section{References}

1. K. Ahmavaara, H. Haverinen, and R. Pichna, "Interworking architecture between 3GPP and WLAN systems," IEEE Commun. Mag., vol.41, no.11, pp.74-81, Nov. 2003.

2. Mobile IP Working Group, "Low Latency Handoffs in Mobile IPv4," draft-ietf-mobileiplowlatency-handoffs-v4-00.txt, Feb. 2001..

3. G. Tsirtsis et al., "Fast Handovers for Mobile IPv6," draft-ietf-mobileip-fast-mipv6-00.txt, Feb. 2001.

4. Amir Majlesi, Babak H. Khalaj, "An Adaptive Fuzzy Logic Based Handoff Algorithm for Interworking between WLANs and Mobile Networks," IEEE PIMRC 2002. 